\title{
THERMAL ANALYSIS OF THE AL WINDOW FOR A NEW CESR-C LUMINOSITY MONITOR*
}

\author{
Y. He\#, Y. Li, M. A. Palmer, D. Rice \\ Laboratory for Elementary-Particle Physics, Cornell University, Ithaca, NY 14853, U.S.A.
}

\section{Abstract}

A luminosity monitor using photons from radiative bhabha events at the CLEO interaction point (IP) has been installed in the Cornell Electron Storage Ring (CESR) [1]. A key vacuum and detector component is the photon window/converter whose uniformity and thickness are critical for determining the resolution of the total energy deposited in the segmented luminosity monitor. The window design must accommodate the operational requirements of the new monitor at CLEO-c beam energies of 1.5-2.5 GeV and also provide sufficient safety margin for operation at $5.3 \mathrm{GeV}$ beam energies for Cornell High Energy Synchrotron Source (CHESS) running. During $5.3 \mathrm{GeV}$ operation, intense stripes of synchrotron radiation (SR) from the interaction region (IR) superconducting quadrupole magnets (SC Quads) as well as nearby bending magnets strike the window. During the course of window development, several materials and designs were considered. Thermal stresses were calculated using ANSYS for various beam conditions to guide the cooling design. A window using aluminum (Al) alloy 6061-T6 was ultimately chosen to provide optimal performance for both CLEO-c and CHESS running conditions. The window has been in successful operation since September 2004.

\section{INTRODUCTION}

The new fast luminosity monitor, which is placed approximately $16.1 \mathrm{~m}$ downstream from the IP in the direction of positron beam is made of an $\mathrm{Al}$ window and a detector. The radiative bhabha photons emitted at the IP travel through the vacuum chamber up to bending magnet $\mathrm{B} 3 \mathrm{~W}$, and exit through the window. The photon rate is measured by counting photons converted in the window. In order to give a clear path for the photons, over 8 meters of CESR vacuum chamber near the IR were modified, providing increased horizontal and vertical aperture [2]. The window is a part of CESR vacuum chamber wall so that the detector can be placed outside the vacuum.

The detector monitors the luminosity in the operation at CLEO-c beam energies of $1.5-2.5 \mathrm{GeV}$ and will be removed for CHESS operation at $5.3 \mathrm{GeV}$. The following window design criteria were considered:

- The window must be thin for two reasons. First, we wanted to be able to operate the detector in a photon counting mode. Secondly, we wanted to keep the radiation dose sufficiently small to use a scintillator-based detector.

- Good conductive and convective cooling to absorb

*Work supported by the US National Science Foundation

\#yh63@cornell.edu
SR power during $5.3 \mathrm{GeV}$ high energy operation. The material should have a good thermal conductivity.

- Window should have a uniform thickness to preserve geometric information from the photon distribution.

\section{WINDOW DESIGN}

We initially considered a $1.5 \mathrm{~mm}$ beryllium $(\mathrm{Be})$ window located at $15 \mathrm{~m}$ from the IP, which provides a horizontal aperture to accept photon beam originated at the IP with crossing angles of the colliding beams ranging from -4.5 to $+2 \mathrm{mrad}$ and a vertical aperture of $-5 / 3 \mathrm{mrad}$ $<y^{\prime}<+5 / 3$ mrad. Because of the high thermal stress and the high cost of fabrication in making the Be window, we then evaluated the design of a $12.7 \mathrm{~mm}$ thick copper window at the same location. Ultimately a window using 6061-T6 Al alloy at the location of $16.1 \mathrm{~m}$ from the IP was chosen which increases the photon beam horizontal acceptance angle to $-7 \mathrm{rmad}<\mathrm{x}^{\prime}<+2 \mathrm{mrad}$, providing a full range photon collection horizontally. It also allows direct welding of the window to the Al chamber HB4W. The vacuum chamber HB4W was modified with a slot with its vertical opening narrowing towards the window, to allow the photons to escape from the ring while still preserving the continuity of the chamber wall as seen by the beam.

The Al window is $25.4 \mathrm{~mm}$ thick, $50.8 \mathrm{~mm}$ high and $110.5 \mathrm{~mm}$ wide. A sandwich structure, shown in figure 1, was selected in order to have a uniform thickness as seen by the FLM photons. A flat water cooling channel is designed to be right behind the SR intercept surface to provide more effective cooling of the window. The keyedin interlayer plate compensates the removed material in the path of photons and enforces channeled water flow.

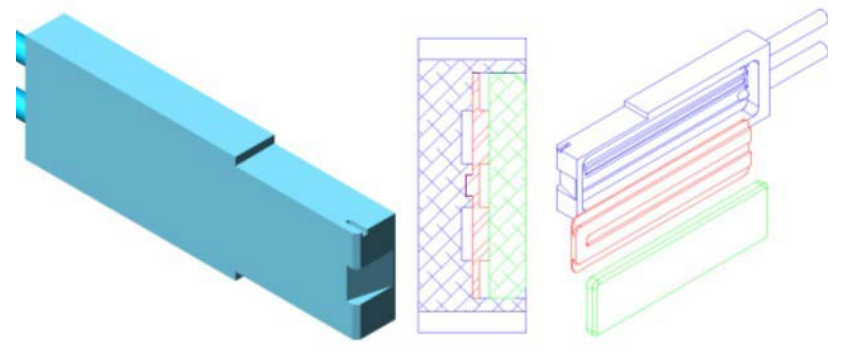

Figure 1: Structure of window

\section{SR POWER IN HIGHER ENERGY OPERATION}

During high energy $5.3 \mathrm{GeV}$ operation, intense stripes of SR from the IR SC Quads (Q1W, Q2W, and PMQ) as well as nearby bending magnets (SB3W and B3W) strike 
the window. The SR power density and distribution from the IR SC Quads depends on positron beam parameters at the IP. We considered two cases with $300 \mathrm{~mA}$ positron beam as a conservative high value. One is in "CHESS nominal lattice" when the positrons have a horizontal displacement of $X=0 \mathrm{~mm}$ and $X^{\prime}=-3.12 \mathrm{mrad}$. The other one is in "Worst Case" when the positrons have a maximum possible horizontal displacement of $X=-4.843$ $\mathrm{mm}$ and $\mathrm{X}^{\prime}=-3.12 \mathrm{mrad}$ at $\mathrm{Q} 2 \mathrm{~W}$. Meanwhile, the SR generated from bending magnets $\mathrm{SB} 3 \mathrm{~W}$ and $\mathrm{B} 3 \mathrm{~W}$ also strikes the window. Since the bending magnets are close to the window, the SR power from SB3W and $\mathrm{B} 3 \mathrm{~W}$ deposited on the window is quite high. Figure 2 shows the power density and distribution in these two conditions.

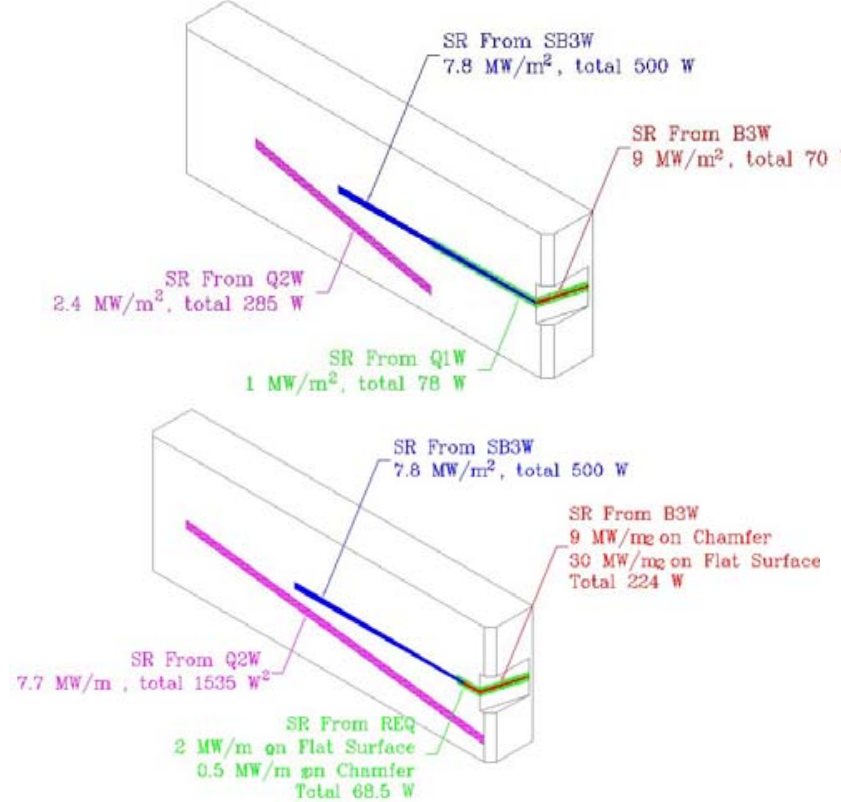

Figure 2: SR power distributions. Top -nominal CHESS lattice; Bottom - Worst case, both with $300 \mathrm{~mA}$ positron

Though a chamfer is made for reducing the power density on the tip of the window, the power density near the chamfered tip is still as high as $30 \mathrm{MW} / \mathrm{m}^{2}$ in the worst case scenario. It raised a concern of the thermal stress induced by such a high power density. Thus thermal analysis of the window was performed using finite element analysis program ANSYS for the two SR power cases shown in figure 2 .

\section{THERMAL ANALYSIS}

The aim of ANSYS simulations is to provide an optimum cooling design, determine the window thickness and determine if the window could survive at $5.3 \mathrm{GeV}$ energy CHESS operation.

A full size 3D model and the following parameters were used for ANSYS calculations:

- Heat flux on the areas shown in figure 2.

- Convection in the water cooling channel with a flow rate of $0.13 \mathrm{l} / \mathrm{s}$, which provides a film coefficient of $4.8 \times 10^{4} \mathrm{~W} / \mathrm{m}^{2} \mathrm{~K}$. Water temperature is $20{ }^{\circ} \mathrm{C}$.
- $27{ }^{\circ} \mathrm{C}$ air cooling around the outer surface of the window, providing a film coefficient of $1.5 \times 10^{2} \mathrm{~W} / \mathrm{m}^{2} \mathrm{~K}$.

- $\mathrm{Al}$ 6061-T6 as the window material.

The results of steady state thermal analysis are shown in figures 3 and 4. In CHESS nominal lattice, the maximum temperature on the tip may reach $91{ }^{\circ} \mathrm{C}$, with the maximum thermal stress of $64 \mathrm{MPa}$ which is $1 / 4 \mathrm{O}$ of $\mathrm{Al}$ tensile yield strength. As for the worst case scenario, the maximum temperature on the tip could be $151{ }^{\circ} \mathrm{C}$, with the maximum thermal stress of $118 \mathrm{MPa}$ which still has a safety factor of 2.4. It should be noted that the Worst Case is an unlikely case for CESR running. Thermocouples are installed to warn the happening of over-heating.

During the operation the window will be subjected to many thermal cycles from beam on-off cycles, so fatigue failure must be considered. With long term exposure at $100^{\circ} \mathrm{C}$, the tensile yield strength of $\mathrm{Al}$ is reduced to $90 \sim$ $95 \%$ of its value at room temperature. ANSYS simulations were based on the positron beam current of $300 \mathrm{~mA}$. In reality CESR operates at lower currents due to many limitations so the window actually experiences less than $91{ }^{\circ} \mathrm{C}$ temperature. Taking into account all of these factors, we conclude that the $\mathrm{Al}$ window design has sufficient thermal safety margin.
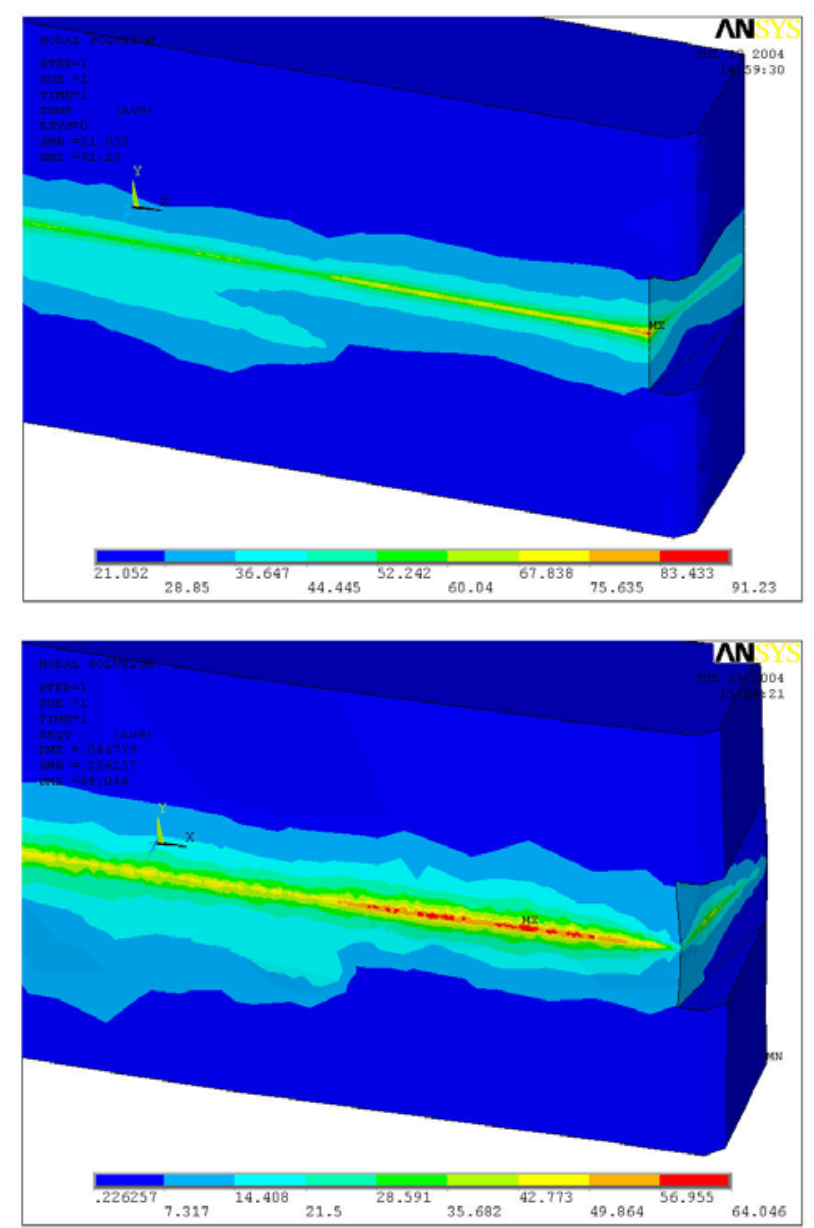

Figure 3: Temperature $\left({ }^{\circ} \mathrm{C}\right.$, top) and thermal stress (MPa, bottom) in CHESS operation. 

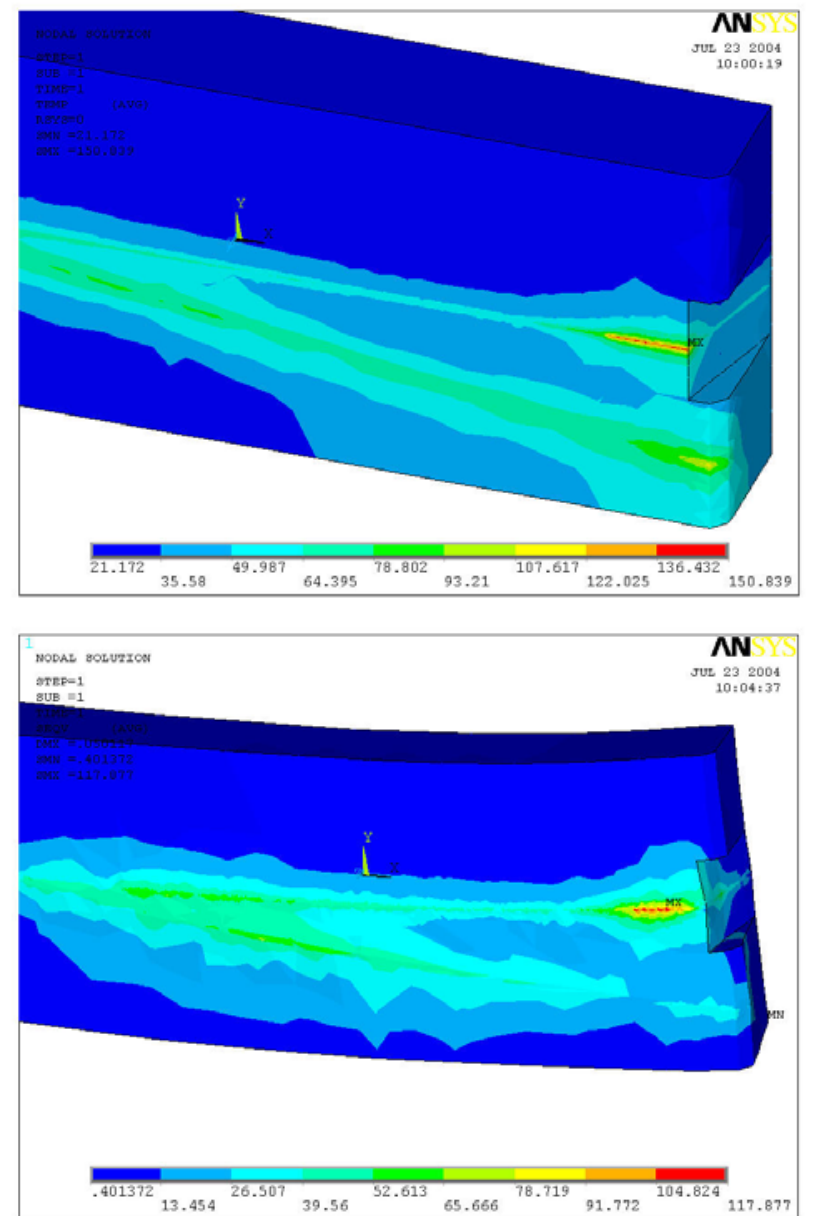

Figure 4: Temperature $\left({ }^{\circ} \mathrm{C}\right.$, top) and thermal stress (MPa, bottom) in the worst case.

\section{OPERATION}

To monitor the temperature of the window, two thermocouples were installed near the chamfered tip. The measured temperature data during CESR operation at low beam current were used to project the temperature at 300 $\mathrm{mA}$. The temperature measurement is in good agreement with the prediction of ANSYS thermal analysis, shown in figure 5 .

\section{CONCLUSION}

An Al window for a new CESR-c fast luminosity monitor has been designed and is in successful operation. The window has sufficient thermal safety margin for high energy operations at CESR. Thermal analysis was critical to the design. Experience during high energy physics operation has demonstrated that the window has sufficient aperture and thickness for operation of the new luminosity monitor.
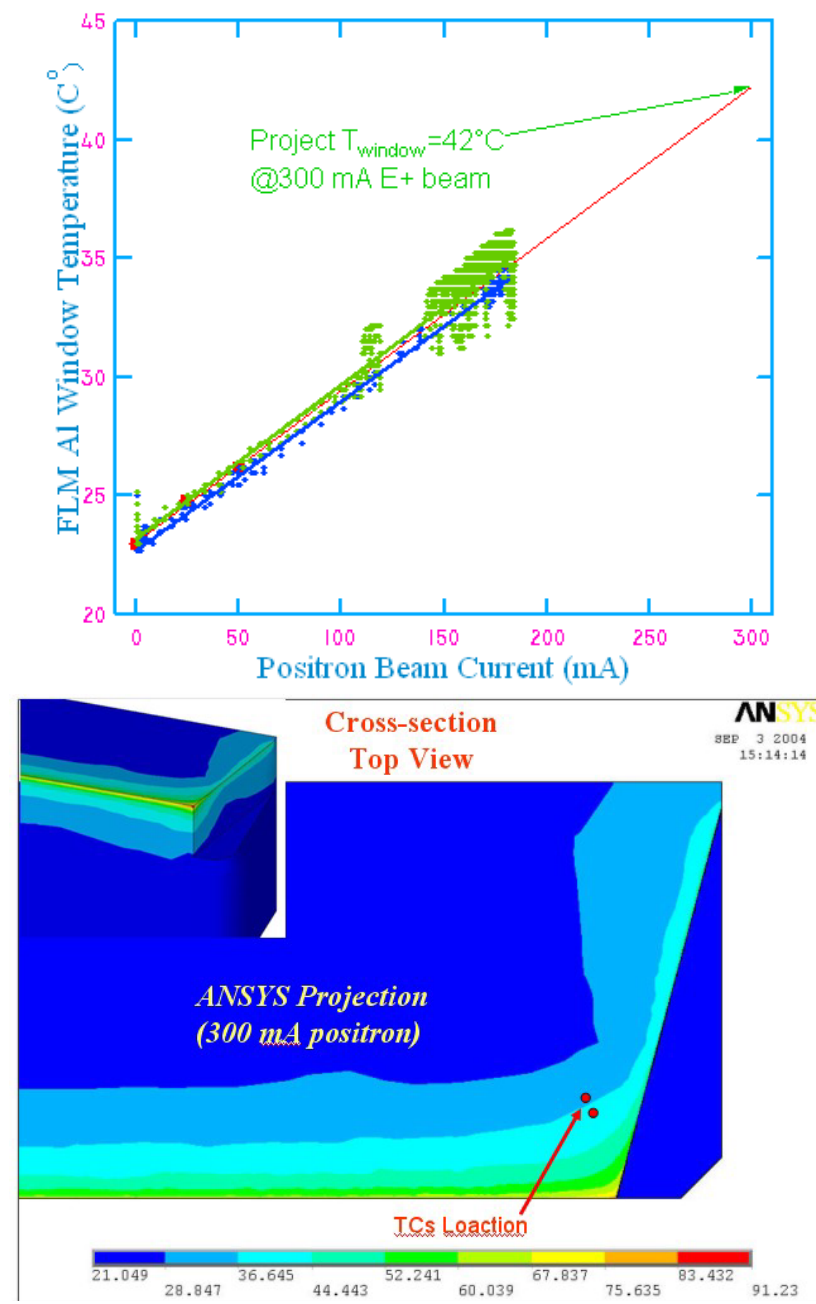

Figure 5: Comparison of the temperature of thermocouple measurement (top) and ANSYS simulation (bottom).

\section{REFERENCES}

[1] M. A. Palmer, et al, "Design and Operation of a Radiative Bhabha Luminosity Monitor for CESR-c", These Proceedings (RPTA062).

[2] Y. Li, Y. He, M. A. Palmer, "Vacuum Modifications for the Installation of a New CESR-c Fast Luminosity Monitor", These Proceedings (RPPE044). 